\title{
Heavy meson production at a low-energy photon collider.
}

\author{
Stephen Asztalos \\ Lawrence Livermore National Laboratory \\ 7500 East Avenue, Livermore, Ca 94551
}

\begin{abstract}
A low-energy $\gamma \gamma$ collider has been discussed in the context of a testbed for a $\gamma \gamma$ interaction region at the Next Linear Collider (NLC). We consider the production of heavy mesons at such a testbed using Compton-backscattered photons and demonstrate that their production rivals or exceeds those by BELLE, BABAR or LEP where they are produced indirectly via virtual $\gamma \gamma$ luminosities.
\end{abstract}

Key words:

UCRL-PROC-203766

\section{Introduction}

Quantum Chromodynamics (QCD) is a gauged theory of the strong interactions. Exquisitely predictive in the perturbative high-energy regime, at present a description of the physical states is beyond the reach of the theory. Meson spectroscopy allows a more complete understanding of QCD in this nonperturbative realm.

Heavy meson spectroscopy provides unique insight into the underlying theory without the additional complications associated with the the lighter (and more relativistic) mesons. Heavy mesons have been studied at $e^{+} e^{-}$colliders for nearly 30 years, however, only selective states compatible with the quantum numbers of the $e^{+} e^{-}$beams are produced directly: other states are populated via subsequent hadronic or electromagnetic decays. Often, as is the case with the $\eta_{b}(\mathrm{nS})$ mesons, the relevant branching ratios are very small. Photon interactions provides a mechanism by which some of these inaccessible mesons

Email address: asztalos1llnl.gov (Stephen Asztalos). 
are produced directly. In a way that is complementary to $e^{+} e^{-}$interactions, $\gamma \gamma$ interactions produce particles having final states with $C$ of +1 , where $C$ is the charge conjugation number.

Participating photons can be either virtual or real. Virtual photons are an irreducible description of charged particle beams: all $\mathrm{e}^{+} \mathrm{e}^{-}$colliders generate virtual $\gamma \gamma$ luminosity - in the case of PEP-II or KeK-II the virtual luminosity can rival the $\mathrm{e}^{+} \mathrm{e}^{-}$luminosity. An alternate mechanism for producing $\gamma \gamma$ luminosity is with real photons. Typically, low energy photons are made to Compton-backscatter off of an energetic charged particle beam to produce highly boosted photons. Opposing photon beams are kinematically focused and interact to produce real $\gamma \gamma$ luminosity. Although this production mechanism requires the added complication of introducing a laser and associated optics into the interaction region, the substantial benefits include control over the $\gamma \gamma$ luminosity profile, polarization and magnitude. Technology advances in optics and lasers have progressed to the point that one could construct a low energy $\gamma \gamma$ testbed as a proof-of-principle for the NLC, where it would be possible to singly produce the Higgs boson (whereas it must be produced in pairs at $\mathrm{e}^{+} \mathrm{e}^{-}$colliders). The potential this offers for doing precision Higgs studies is a major driving force behind the impetus for a $\gamma \gamma$ option at the NLC. For the purposes of this study, however, we restrict our attention to photons with a center of mass energy of $60 \mathrm{GeV}$, which is sufficient for an engineering demonstration. One possible site for the testbed is the Stanford Linear Collider, where polarized electron beams meet opposing positrons beams at center-of-mass energies up to the mass of the $\mathrm{Z}_{0}$. In this proceeding we demonstrate that with suitable choice of laser and optics parameters the resulting $\gamma \gamma$ luminosity can be made to exceed the virtual $\gamma \gamma$ luminosity from $\mathrm{e}^{+} \mathrm{e}^{-}$colliders. This luminosity can thus serve as the basis for a physics program at the testbed facility, e.g., heavy quarkonia studies. In subsequent sections we compare and contrast real and virtual $\gamma \gamma$ luminosities and discuss the implications for heavy quark meson spectroscopy.

\section{Real photon luminosity}

Depending on the energy transfer scale the structure of an otherwise featureless photon can be resolved, resulting in an irreducible hadron and leptonic background that would complicate precision Higgs measurements. Hence, a primary goal of a $\gamma \gamma$ testbed would be to measure the $\gamma \gamma$ luminosity and compare the result with simulation. In this study Compton-backscattered luminosity was simulated with CAIN 2.1e (1) using the laser and beam parameters listed in Table 1, where the electron and laser polarizations refer to circular polarization. Beam parameters in this table reflect NLC-like expectations about achievable beam sizes, while the laser parameters were chosen to maxi- 
Table 1

\begin{tabular}{|c|c||c|c|}
\hline \multicolumn{2}{|c||}{ Beam } & \multicolumn{2}{c|}{ Laser } \\
\hline parameter & value & parameter & value \\
\hline \hline beam energy & $30 \mathrm{GeV}$ & frequency & $120 \mathrm{~Hz}$ \\
\hline charge & $4 \times 10^{10}$ & pulse energy & $2.0 \mathrm{~J}$ \\
\hline$\sigma_{x}$ & $1.47 \mu \mathrm{m}$ & $\lambda$ & $1.05 \mu \mathrm{m}$ \\
\hline$\sigma_{y}$ & $0.0552 \mu \mathrm{m}$ & laser polarization & \pm 1 \\
\hline$\sigma_{z}$ & $0.1 \mathrm{~mm}$ & & \\
\hline$\beta_{x}$ & $8.0 \mathrm{~mm}$ & & \\
\hline$\beta_{y}$ & $0.1 \mathrm{~mm}$ & & \\
\hline$e^{-}$polarization & 0.8 & & \\
\hline$e^{+}$polarization & 0 & & \\
\hline
\end{tabular}

Beam and laser parameters used to characterize the real photon luminosity at LINX.

mize the Compton-backscattering rate. (Throughout this proceeding we refer to a hypothetical accelerator whose beam parameters match those of Table 1 as LINX.) Figure 1 shows the resulting simulated Compton-backscattered electron and resultant photon spectra at the LINX conversion point. With nearly $2 \times 10^{10}$ photons per electron the conversion is very efficient: more than $98 \%$ of the electrons undergo Compton-scattering. The preponderance of photons also causes significant degradation of the single-interaction photon and electron Compton edges around 10 and $20 \mathrm{GeV}$, respectively. This degradation is due to multiple Compton interactions as the electron beam traverses the

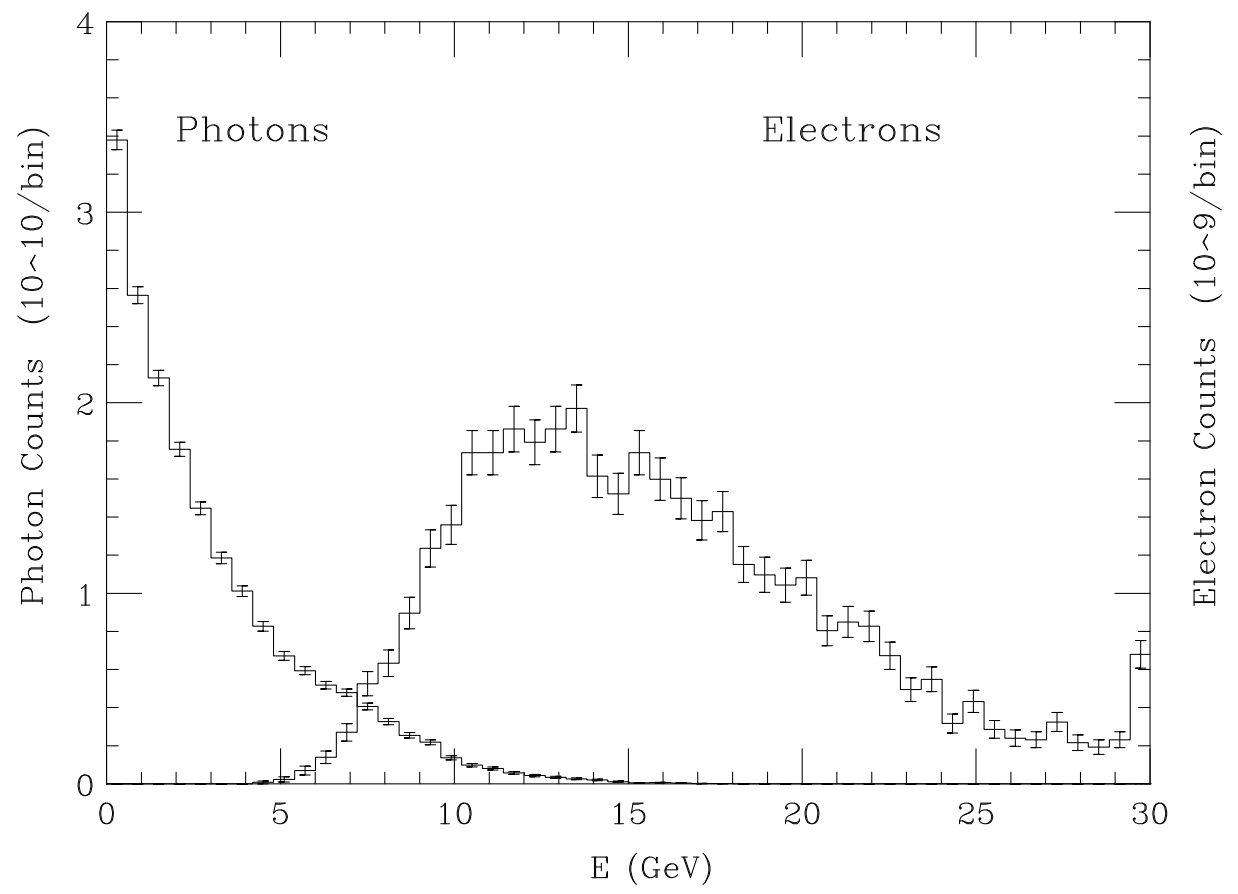

Fig. 1. A snapshot of the right-going photons and electrons at the conversion point. The ratio of backscattered photons to initial electrons is approximately $4: 1$. Note the different axis scalings for photon and electrons. 
laser pulse. This picture is quite distinct from the sharp Compton edges seen in simulations (not shown) where the energy of the laser pulse is considerably lower and single interactions dominate.

$\gamma \gamma$ luminosity is produced when the left- and right-going photons traverse the several millimeters from their respective conversion points to the interaction point. Figure 2 shows the resulting simulated Compton-backscattered $\gamma \gamma$ luminosity at the interaction point from CAIN. For this analysis the luminosity is divided into 50 equally spaced energy bins spanning the range $0<\sqrt{s}<$ $62.4 \mathrm{GeV}$. Multiple Compton interactions give rise to significant luminosity

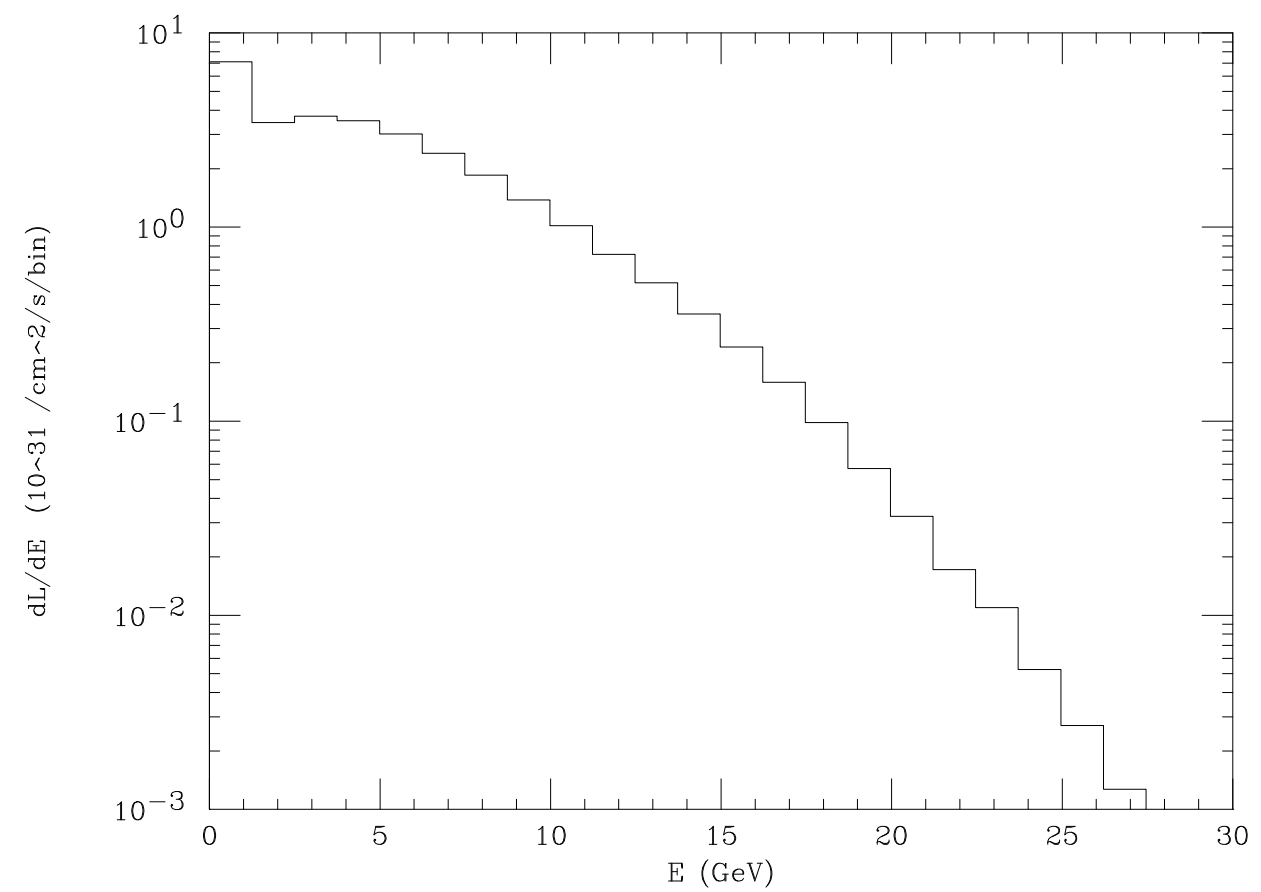

Fig. 2. $\gamma \gamma$ luminosity for the beam and laser parameters in Table 1. The total integrated (instantaneous) luminosity is $2.971 \times 10^{32} \mathrm{~cm}^{-2} \mathrm{~s}^{-1}$

above $20 \mathrm{GeV}$, whereas for lower laser energies (where single interactions dominate) the luminosity is essentially zero for energies above twice the Compton edge. The area under the curve in Fig. 2, or the instantaneous $\gamma \gamma$ luminosity, is $2.971 \times 10^{32} \mathrm{~cm}^{-2} \mathrm{~s}^{-1}$. By way of comparison, the maximum instantaneous $\mathrm{e}^{+} \mathrm{e}^{-}$luminosity at LEP never exceeded $1.0 \times 10^{32} \mathrm{~cm}^{-2} \mathrm{~S}^{-1}$ over its $10+$ years of operation.

\section{$3 \quad$ Virtual photon luminosity}

To assess the relative merits of a real photon collider we have considered three colliders whose large $\mathrm{e}^{+} \mathrm{e}^{-}$luminosities would suggest a large virtual 
$\gamma \gamma$ luminosity: PEP-II (2), KEK-B (3) and LEP (4). PEP-II and KEK-B are asymmetric $e^{+} e^{-}$colliders running at a center of mass energy of $10.58 \mathrm{GeV}$, while LEP was a symmetric $e^{+} e^{-}$machine that scanned over the energy range $91.2<\sqrt{s} \lesssim 206 \mathrm{GeV}$ until decommissioned in late 2000 to make way for the Large Hadron Collider (LHC).

The classical treatment of virtual photons exploits the equivalence between the fields of energetic charged particles and pulses of electromagnetic radiation. In the equivalent photon approximation (EPA) a pulse of electromagnetic radiation is transformed into a frequency spectrum of virtual photons. The interaction of virtual photons from colliding charged particle beams gives rise to virtual photon luminosity. The Weizsacker Williams approximation to the EPA restricts itself to transverse modes of the virtual photon while integrating over the (unobserved) momentum transfer between the electron and the virtual photon to yield (5)

$$
\frac{d N}{d z}=\frac{\alpha}{2 \pi z}\left[\left(1-z+\frac{z^{2}}{2}\right) \log \left(\frac{q_{\max }^{2}}{q_{\min }^{2}}\right)-2 m_{e}^{2}\left(\frac{1}{q_{\min }^{2}}-\frac{1}{q_{\max }^{2}}\right) z^{2}\right]
$$

where $z$ is defined as $\mathrm{E}_{\gamma} / \mathrm{E}_{\text {beam }}, m_{e}$ the electron (or positron) mass, $q_{m i n}^{2}$ and $q_{\text {max }}^{2}$ are the minimum and maximum photon energies, respectively, and $d N / d z$ is the number of photons in an energy interval $d z$. Both $q_{\min }^{2}$ and $q_{\max }^{2}$ depend on $z$ in the following manner

$$
\begin{aligned}
q_{\min }^{2} & =\frac{m_{e}^{2} z^{2}}{1-z} \\
q_{\text {max }}^{2} & =(1-z) E_{\text {beam }}^{2} 2\left(1-\cos \theta_{\text {max }}\right)
\end{aligned}
$$

The dependence of $q_{\max }^{2}$ on $\theta_{\max }$ in Eq. 3 merits comment. As $\theta_{\max }$ increases the opportunity for detecting electrons diminishes due to limited detector acceptance, but it is precisely these unobserved electrons that contribute to Eq. 1. The appearance of $\theta_{\text {max }}$ the above expression requires that specific detector configurations be taken into account in the WW analyses. BABAR (6) and BELLE (7) detectors surround the interaction regions at PEP-II and KEK-B, respectively. We take the OPAL (4) detector as the prototypical LEP detector, while the acceptances of the LINX detector were assumed to be those of the

Table 2

\begin{tabular}{|c|c|c|c|c|}
\hline & BABAR & BELLE & OPAL & LINX \\
\hline Energy $(\mathrm{GeV})$ & $3.1 / 9.0$ & $3.5 / 8.0$ & $91-206 / 91-206$ & $30 / 30$ \\
\hline$\theta_{\max }(\mathrm{mrad})$ & $523.6 / 295.6$ & $715.6 / 401.4$ & $33 / 33$ & $36 / 36$ \\
\hline
\end{tabular}

$e^{+} / e^{-}$beam energies and forward/backward acceptances for the BABAR, BELLE, OPAL and LINX detectors. 
Table 3

\begin{tabular}{|c|c|c|c|c|c|c|}
\hline \multirow{2}{*}{ year } & \multicolumn{2}{|c|}{ BABAR } & \multicolumn{2}{c|}{ BELLE } & \multicolumn{2}{c|}{ OPAL } \\
\cline { 2 - 7 } & peak $^{1}$ & int $^{2}$ & peak & int & peak & int \\
\hline \hline 1990 & - & - & - & - & $0.0110^{3}$ & 0.0121 \\
1991 & - & - & - & - & $0.0110^{3}$ & 0.0189 \\
1992 & - & - & - & - & $0.0110^{3}$ & 0.0286 \\
1993 & - & - & - & - & 0.0190 & 0.0400 \\
1994 & - & - & - & - & 0.0231 & 0.0645 \\
1995 & - & - & - & - & 0.0341 & 0.0461 \\
1996 & - & - & - & - & 0.0356 & 0.0247 \\
1997 & - & - & - & - & 0.0570 & 0.0734 \\
1998 & - & - & - & - & 0.0999 & 0.1997 \\
1999 & 1 & 1.62 & $2.05^{4}$ & 0.287 & 0.1069 & 0.2537 \\
2000 & 2 & 23.76 & 2.05 & 10.940 & 0.0675 & 0.2331 \\
2001 & 4.4 & 40.05 & 5.171 & 36.257 & - & - \\
2002 & 5 & 31.32 & 8.256 & 54.181 & - & - \\
2003 & 7.5 & 56.71 & 11.305 & 77.232 & - & - \\
2004 & $7.5^{5}$ & 4.63 & $11.305^{5}$ & 6.827 & - & - \\
\hline \hline Total & - & 158.09 & & 185.724 & - & 0.9948 \\
\hline
\end{tabular}

Instantaneous and integrated $e^{+} e^{-}$luminosities as a function of year. For BABAR and BELLE the 2004 luminosities are through 01/04.

Stanford Linear Detector (8). Table 2 lists the beam energies and forward and backward acceptances seen by the four detectors under consideration. The forward and backward acceptances of BABAR and BELLE are unequal due to the asymmetric beam energies.

In order to compare real and virtual $\gamma \gamma$ luminosities yearly $\mathrm{e}^{+} \mathrm{e}^{-}$luminosity records for BABAR, BELLE and OPAL were compiled. Table 3 summarizes the peak and integrated $e^{+} e^{-}$luminosities seen by each detector since initiation of their respective operations.

\section{Results}

To simulate Weizsacker Williams luminosity the physics event generator PANDORA 2.1 (9) was used. Since Eq. 1 increases without limit as $\mathrm{z} \rightarrow 0$, it was necessary to introduce a low-energy cutoff to regularize the result. An energy cutoff of $1 \mathrm{MeV}$ was employed in all subsequent analyses. The choice of cutoff obviously affects the integrated virtual luminosity, but has no discernible

\footnotetext{
$\overline{1 \text { In units of } n b^{-1} \mathrm{~s}^{-1}}$

2 In units of $\mathrm{fb}^{-1}$

3 LEP peak luminosities assumed for 1990-1992.

4 BELLE peak luminosity not available for 1999.

5 BELLE and BABAR peak luminosities for 2004 taken from 2003.
} 
affect on the virtual photon spectra above the energy range of interest $(\sim 3$ $\mathrm{GeV}$ ), nor on the luminosity integrated cross sections discussed below. Virtual $\gamma \gamma$ luminosity was calculated for the PEP-II, KEK-B and LEP beams using Pandora in two steps. First, a random number corresponding to the energy of a virtual in one beam was generated. Associated with this photon energy is a specific value of $d N / d z$ from the Weizsacker Williams approximation. This same procedure was repeated for the opposing beam. The product of the $d N / d z$ from each opposing beam gives the relative virtual $\gamma \gamma$ luminosity per unit $e^{+} e^{-}$luminosity, which for PEP-II was determined to be 0.19 . Instantaneous virtual $\gamma \gamma$ luminosities were derived by multiplying the relative virtual $\gamma \gamma$ luminosities by the instantaneous $e^{+} e^{-}$luminosities from Table 3. Although the table contains yearly peak luminosities (when available), for our purpose the peak luminosity with the greatest magnitude was used. A comparison with the LINX instantaneous $\gamma \gamma$ luminosity is shown in the lower graph in Fig. 3. In both graphs in this figure and in the analyses described below the BELLE and BABAR luminosities have been summed together, while the LEP luminosity is that seen by the OPAL detector, but multiplied by four to account for the four LEP detectors. The characteristic rise inherent in the Weizsacker

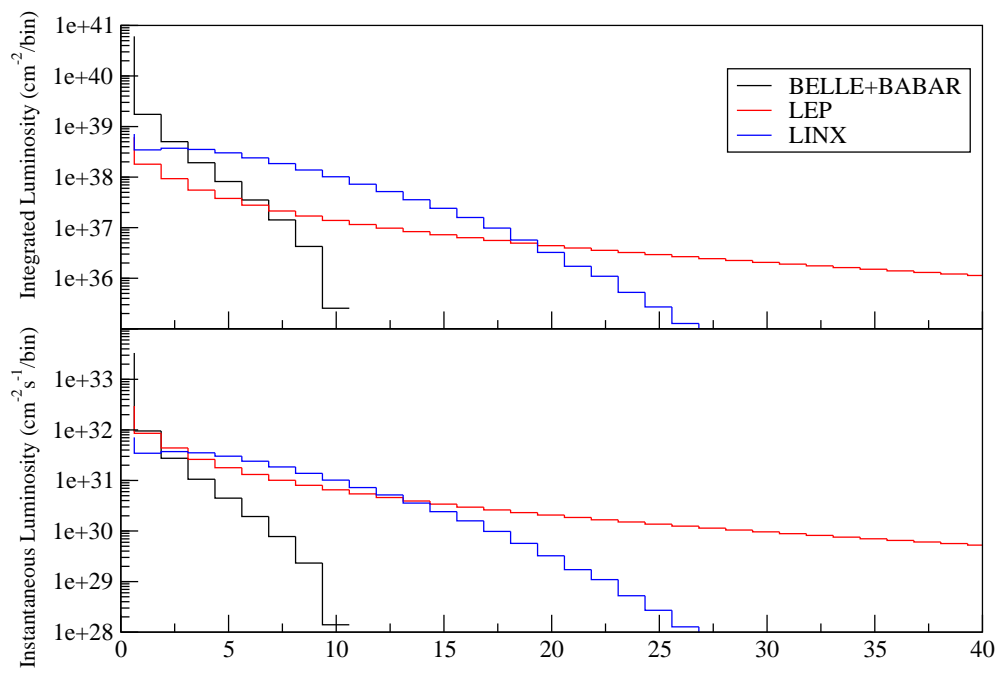

Fig. 3. The instantaneous (lower) and integrated (upper) virtual Weizsacker-Williams luminosities seen by BELLE+BABAR and LEP compared to the real luminosity seen at LINX. All luminosities are in $1.3 \mathrm{GeV}$ bins.

Williams description of virtual photons is seen in the BELLE+BABAR and LEP plots, whereas the rise in the LINX luminosity at low energies stems instead from bremsstrahlung and multiple scattering. Using the relative $\gamma \gamma$ luminosities above and the peak luminosity data from Table 3 , one arrives at peak instantaneous luminosities of $3.479 \times 10^{33} \mathrm{~cm}^{-2} \mathrm{~s}^{-1}$ and $3.121 \times 10^{32} \mathrm{~cm}^{-2} \mathrm{~s}^{-1}$ for BABAR+BELLE and LEP, respectively. By comparison, the LINX instantaneous luminosity is $2.971 \times 10^{32} \mathrm{~cm}^{-2} \mathrm{~s}^{-1}$. 
Table 4

\begin{tabular}{|l|c|c|c|}
\hline & BABAR+BELLE & LEP & LINX \\
\hline norm & 0.19 & $0.52-0.70$ & 1.0 \\
\hline inst $\left(10^{32} \mathrm{~cm}^{-2} \mathrm{~s}^{-1}\right)$ & 34.79 & 3.121 & 2.971 \\
\hline intr $\left(10^{39} \mathrm{~cm}^{-2} \mathrm{~s}\right)$ & 63.86 & 2.60 & 2.971 \\
\hline
\end{tabular}

Normalized, instantaneous and integrated luminosities from BABAR+BELLE, LEP and LINX. One Snowmass year of operation $\left(10^{7} \mathrm{~s}\right)$ was assumed for the LINX integrated luminosity.

A similar approach was used in producing the integrated luminosities in the top graph in Fig. 3, but here the relative virtual $\gamma \gamma$ luminosities are multiplied by the yearly integrated luminosities and summed over relevant years to form a total integrated luminosity. For instance, in the case of LEP there are 11 contributions to the sum (years 1990 to 2000) - each year with its own relative and integrated luminosity to account for the changing beam energy with time. A summary of the results for the normalized, instantaneous and integrated luminosities is contained in Table 4 . Taken together, Table 4 and the top graph in Fig. 3 is one of the our main findings: although the integrated virtual $\gamma \gamma$ luminosity from BELLE+BABAR greatly exceeds the integrated real $\gamma \gamma$ luminosity expected from LINX, due to the sharply decreasing nature of the Weizsacker-Williams spectrum this dominance disappears beyond $3.0 \mathrm{GeV}$. As will be seen in the next section, since much of the physics of interest lies above this energy, real $\gamma \gamma$ luminosity offers a very viable approach to heavy meson spectroscopy.

\section{Physics Opportunities}

One goal of heavy quark spectroscopy is to test Quantum Chromodynamics predictions of the masses, widths and quantum numbers of these mesons by comparing them with their experimental values. Production of pseudoscalar mesons is a particularly attractive physics goal for a low-energy $\gamma \gamma$ collider since the mesons quantum numbers $\mathrm{J}^{C P}=0^{+-}$preclude them from being produced directly at an $e^{+} e^{-}$collider. Although the lowest radial excitations of the charmonium system has been thoroughly mapped out (with the exception of the singlet $h(1 \mathrm{p})$ state), spectroscopy of the second radial excitation has numerous holes. Even where there have been recent measurements, as in the case of the pseudoscalar $\eta_{c}(2 \mathrm{~S})$, disagreement persists as to the excitation (mass). Conflict between experiment and theory on the order of $50 \mathrm{MeV}$ in mass of the $\eta_{c}(2 \mathrm{~S})$ is the source of some consternation in the theoretical community (10). Enhanced statistics would further allow for the determination of unknown branching ratios. The situation is more severe in the bottom system: none of the pseudoscalar bottom mesons have been definitively observed (11). Bottom pseudoscalar mesons apparently are more difficult to produce at $e^{+} e^{-}$ colliders: their heavier heavier masses imply a more non-relativistic wave func- 


\begin{tabular}{|c||c|c|c|}
\hline meson & mass & $\Gamma_{\text {tot }}$ & $\Gamma_{\gamma \gamma}$ \\
\hline \hline$\eta_{c}(1 \mathrm{~S})$ & 2.979 & 0.0161 & $7.4 \times 10^{-6}$ \\
\hline$\eta_{c}(2 \mathrm{~S})$ & 3.654 & 0.0161 & $7.4 \times 10^{-6}$ \\
\hline$\chi_{c 0}$ & 3.415 & 0.0107 & $2.6 \times 10^{-6}$ \\
\hline$\eta_{b}(1 \mathrm{~S})$ & 9.3 & 0.014 & $5.3 \times 10^{-7}$ \\
\hline
\end{tabular}

Table 5

Masses and widths of the mesons in $\mathrm{GeV}$.

tion. This, in turn, enhances the selection rule which in the non-relativistic limit forbids $E(1)$ transitions between the $\Upsilon(\mathrm{nS}) \rightarrow \eta_{b}(\mathrm{nS})$.

Using the integrated luminosities described in the previous section we have studied the production of pseudoscalar and other suitable heavy charm and bottom mesons at a low-energy $\gamma \gamma$ testbed and have compared these results to what can be achieved through virtual $\gamma \gamma$ luminosity. Meson simulations were again carried out using the PANDORA event generator. Meson production in PANDORA is modeled using the Breit-Wigner approach; necessary modifications were made to include the widths and masses of the various mesons that are summarized in Table 5. To model meson production at LINX CAIN 2.1 files were imported into PANDORA. These CAIN luminosity files have 10000 entries representing the partitioning of the energy range $0<\sqrt{s}<62.4$ $\mathrm{GeV}$ into 50 equally spaced energy bins of $1.248 \mathrm{GeV}$ each for $\mathrm{E}_{\gamma 1}$ and $\mathrm{E}_{\gamma 2}$. There are four sets of 2500 entries corresponding to the four helicity combinations possible when two photons interact. Each of the 10000 entries contains $\mathrm{E}_{\gamma 1}$ and $\mathrm{E}_{\gamma 2}$, luminosity and overall helicity. PANDORA normalizes the luminosity so that the sum over all 10000 entries is 1.0. Meson production at BABAR+BELLE and LEP was modeled using the luminosity class described in Section 3.

PANDORA can be made to output luminosity integrated cross sections for the process of interest, defined as

$$
\bar{\sigma} \equiv \int \frac{\partial L}{\partial E} \sigma d E
$$

In practice, the integral is replaced by a finite sum sampled from a uniformly distributed $\mathrm{E}_{\gamma 1}$ and $\mathrm{E}_{\gamma 2}$ plane. The utility of the luminosity integrated cross section is that when multiplied by the total integrated luminosity the result is the number of events for the process under consideration. Table 6 lists the luminosity integrated cross sections and total number of events produced for each meson in the previous table. Note that unlike the conventional definition of cross section, the luminosity integrated cross section depends on details of the environment in which the meson is produced: it is lowest for BABAR+BELLE, for which the integrated luminosity is greatest. From this same table it is seen that number of $\eta_{c}(1 \mathrm{~S})$ events produced at BABAR+BELLE and LINX are approximately equal. Figure 3 provides 
a consistency check on this result. In the upper plot in that figure the integrated luminosities of BABAR+BELLE and that of LINX are seen to be approximately equal at an energy corresponding to the mass of the $\eta_{c}(1 \mathrm{~S})$. Beyond this crossover point, a greater number of heavier mesons are produced at the LINX facility than at BELLE+BABAR or LEP. For the purposes of bottom spectroscopy the LINX scenario is clearly superior, with the potential for producing an order of magnitude or more $\eta_{b}(1 \mathrm{~S})$ mesons than either BELLE+BABAR or LEP.

In order to simulate experimental reconstruction of these mesons at a $\gamma \gamma$ testbed it is necessary to specify the decay mode, as well as potential backgrounds. Decay of heavy mesons into p $\bar{p}$ pairs was chosen due to the simplicity of the event reconstruction, the relatively large branching ratios and knowledge about the competing background process. The dominant background $\gamma \gamma \rightarrow \mathrm{p} \overline{\mathrm{p}}$ arises when both photons are resolved into hadrons. This process has been well-studied for insight into the photon structure function and resolution of the ongoing debate concerning diquark versus independent quark models of the hadron. Theoretical (12) and experimental (13) efforts have gone into determining the cross section for this process. In particular, the latest BELLE results describe a differential cross section (in $\mathrm{nb} / \mathrm{sr}$ ) for this process of the form

$$
\frac{d \sigma}{d E d(\cos \theta)}=1.15 \times 10^{6} \sigma^{15}\left(\frac{1+\cos ^{2} \theta}{1-\cos ^{2} \theta}\right)
$$

for $|\cos (\theta)| \leq 0.6$ and $\sqrt{s} \geq 2.75 \mathrm{GeV}$. This result is consistent with the diquark model, in contrast to the independent quark model predicts an isotropic angular distribution. The normalization factor that appears in Eq. 5 was derived directly from BELLE data. A separate Pandora class was written to generate the $\gamma \gamma \rightarrow \mathrm{p} \overline{\mathrm{p}}$ background; when convolved with the LINX luminosity described above a luminosity integrated cross section subject to the above kinematic limits of $0.0058 \mathrm{nb}$ is obtained, which corresponds to 17337 events over a Snowmass year of operation. Similarly, to compute the number reconstructed meson events it was necessary to multiply the raw number of meson events from the last row of Table 6 by the branching ratio for decay into $\mathrm{p} \overline{\mathrm{p}}$ pairs. Table 7 summarizes the number of events expected from this proce-

\begin{tabular}{|c|c|c|c|c|c|c|}
\hline \multirow{2}{*}{ meson } & \multicolumn{2}{|c|}{ BABAR+BELLE } & \multicolumn{2}{c|}{ LEP } & \multicolumn{2}{c|}{ LINX } \\
\cline { 2 - 7 } & $\bar{\sigma}$ & events & $\bar{\sigma}$ & events & $\bar{\sigma}$ & events \\
\hline$\eta_{c}(1 \mathrm{~S})$ & $3.19 \mathrm{e}+4$ & $1.08 \mathrm{e}+7$ & $1.72 \mathrm{e}+6$ & $1.98 \mathrm{e}+6$ & $3.34 \mathrm{e}+6$ & $9.91 \mathrm{e}+6$ \\
\hline$\eta_{c}(2 \mathrm{~S})$ & $1.25 \mathrm{e}+4$ & $4.25 \mathrm{e}+6$ & $8.43 \mathrm{e}+5$ & $9.74 \mathrm{e}+5$ & $1.97 \mathrm{e}+6$ & $5.84 \mathrm{e}+6$ \\
\hline$\chi_{c 0}$ & $5.23 \mathrm{e}+3$ & $1.78 \mathrm{e}+6$ & $3.32 \mathrm{e}+5$ & $3.83 \mathrm{e}+5$ & $7.66 \mathrm{e}+5$ & $2.28 \mathrm{e}+6$ \\
\hline$\eta_{b}(1 \mathrm{~S})$ & $3.05 \mathrm{e}-1$ & $1.09 \mathrm{e}+2$ & $3.25 \mathrm{e}+2$ & $3.89 \mathrm{e}+2$ & $1.07 \mathrm{e}+3$ & $3.17 \mathrm{e}+3$ \\
\hline
\end{tabular}

Table 6

The luminosity integrated cross sections and events produced for the three machine scenarios considered. 
dure. The Phythia interface to Pandora allows events to be written in StdHeP

Table 7

\begin{tabular}{|c||c|c|c|}
\hline meson & BR $(\mathrm{p} \overline{\mathrm{p}})$ & $\mathrm{p} \overline{\mathrm{p}}$ & Reconstructed $\mathrm{p} \overline{\mathrm{p}}$ events \\
\hline \hline$\eta_{c}(1 \mathrm{~S})$ & 0.0012 & $1.19 \mathrm{e}+4$ & $7.09 \mathrm{e}+3$ \\
\hline$\eta_{c}(2 \mathrm{~S})$ & 0.00049 & $2.80 \mathrm{e}+3$ & $1.69 \mathrm{e}+3$ \\
\hline$\chi_{c 0}$ & 0.00024 & $5.47 \mathrm{e}+2$ & $3.32 \mathrm{e}+2$ \\
\hline$\eta_{b}(1 \mathrm{~S})$ & 0.0012 & $4.00 \mathrm{e}+0$ & $3.00 \mathrm{e}+0$ \\
\hline \hline$\gamma \gamma \rightarrow \mathrm{p} \overline{\mathrm{p}}$ & - & $1.73 \mathrm{e}+4$ & $1.73 \mathrm{e}+4$ \\
\hline \hline Total & - & $3.26 \mathrm{e}+4$ & $2.64 \mathrm{e}+4$ \\
\hline
\end{tabular}

Branching ratios into $\mathrm{p} \overline{\mathrm{p}}$ final states and total number of signal and background events expected for one Snowmass year of running at a $\gamma \gamma$ testbed.

format (14), which in turn are read in by the LCDROOT (15) - a detector simulation and analysis framework based on ROOT (16). In this analysis the $\mathrm{p} \overline{\mathrm{p}}$ final states and background were propagated through a small version of a hypothetical NLC detector whose electromagnetic and hadronic calorimeters reside in $5 \mathrm{~T}$ magnetic field. Details of the detector (17) are not crucial to the results. Passage of $\mathrm{p} \overline{\mathrm{p}}$ through the detector simulation results in momenta smearing. In the hadronic calorimeter the momenta is inferred. For this analysis only inclusive decays to $\mathrm{p} \overline{\mathrm{p}}$ are considered, thus all particles reaching the hadronic calorimeters were assumed to have the mass of a proton. With this information and the total energy measured in the electromagnetic calorimeter the four vector is constructed and the invariant mass is calculated. Figure 4 is
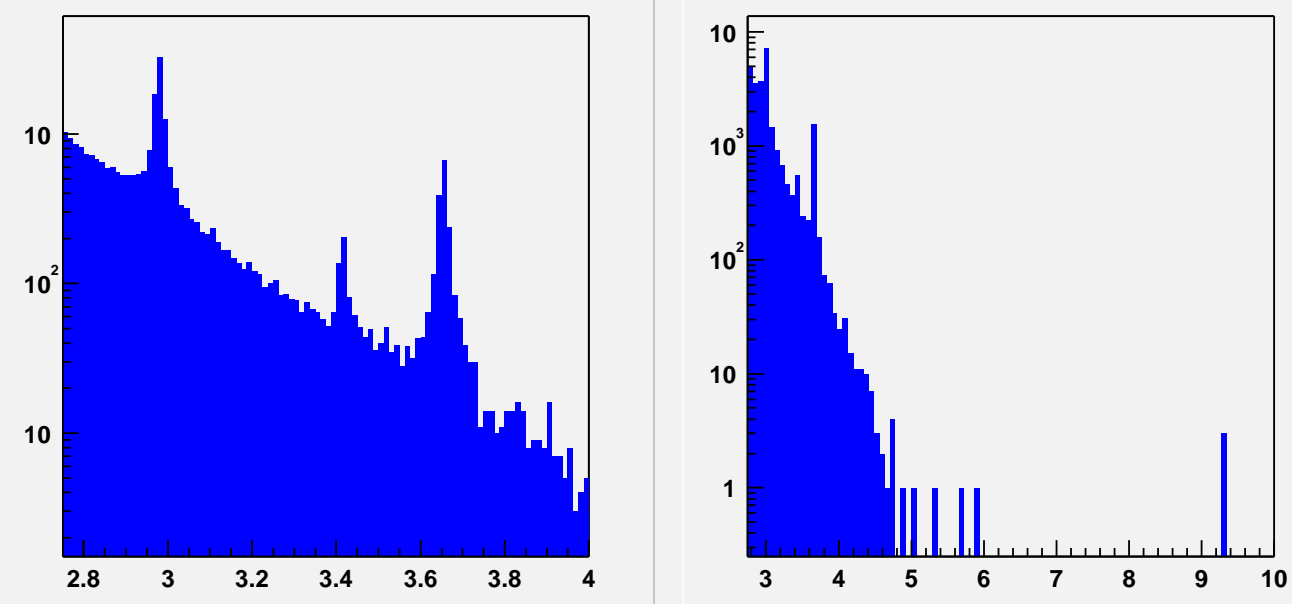

Fig. 4. Reconstructed meson and background events expected from a Snowmass year of operation at LINX. Lower and upper plots are identical except for the scaling of the axes. Cuts of $|\cos (\theta)| \leq 0.6$ and $\sqrt{s} \geq 2.75 \mathrm{GeV}$ were applied to all data.

a reconstruction of the invariant mass of events leaving a track in the hadronic calorimeter subject to the restriction that $|\cos (\theta)| \leq 0.6$ and $\sqrt{s} \geq 2.75 \mathrm{GeV}$. The total number of reconstructed events $(26,494)$ found in the last column of 7 is significantly fewer than the 32,527 StdHeP events input into LCDROOT. 
This is an artifact stemming from the fact that kinematic cuts were not imposed on the meson events within PANDORA but rather inside LCDROOT. For the isotropically decaying mesons the fraction lost is consistent with the restricted phase space imposed by the angular cuts. The meson events clearly stand out above the $\mathrm{p} \overline{\mathrm{p}}$ background, although additional analysis is needed to determine whether it is possible to separate out $\chi_{c 0}$ and $\eta_{c}(2 \mathrm{~S})$ events. A thorough description of these results, including a complete treatment of the non-isotropic decay of the $\chi_{c 2}$, will appear elsewhere.

\section{Conclusions}

A $\gamma \gamma$ interaction region has been proposed for the NLC using Comptonbackscattered photons. Technical challenges integrating the optical, laser and mechanical subsystems suggest the desirability of a low-energy testbed. In this paper we address one aspect of a physics program that could accompany such a test bed - heavy meson production - and compare it with similar meson production expected where virtual photons are the means of production. We find that after a single year of operation the number of charmed mesons seen at a LINX facility equals or exceeds the number generated at either BABAR+BELLE or LEP to-date. LINX is especially dominant when producing bottom mesons, offering the exciting possibility of discovering $\eta_{b}(\mathrm{nS})$ mesons before the LHC begins delivering data toward the end of this decade.

\section{Acknowledgments}

The authors would like to thank Prof. Robert Nisius for helpful insight into the virtual photon methodology.

\section{References}

[1] http://www-acc-theory.kek.jp/members/cain/

[2] http://www.slac.stanford.edu/accel/pepii/home.html

[3] http://belle.kek.jp/

[4] http://opal.web.cern.ch/Opal/

[5] K. F. von Weizsacker, Z. Physik 88, 612 (1934), E. J. Williams, Phys. Rev. 45, (1934) 729.

[6] http://www.slac.stanford.edu/BFROOT/

[7] http://belle.kek.jp/

[8] http://www-sld.slac.stanford.edu/sldwww/sld.html 
[9] http://www-sldnt.slac.stanford.edu/nld/new/Docs/Generators/PANDORA.htm

[10] DELPHI Collaboration, J. Abdallah, et al, Eur. Phys.J. C31 (2003) 481

[11] ALEPH Collaboration, Phys.Lett. B530, (2002) 56

[12] V. M. Budnev et al., Phys. Rep. 15, (1975) 181

[13] Ch.-Ch. Kuo et al., Nucl. Phys.B (Proc. Suppl.) 126, (2004) 313

[14] http://www-cpd.fnal.gov/psm/stdhep/

[15] http://www-sldnt.slac.stanford.edu/nld/New/Docs/LCD_Root/root.htm

[16] http://root.cern.ch/

[17] http://wwwal.kuicr.kyoto-u.ac.jp/www/accelerator/sspm/Iwasaki.pdf 